\title{
Impacts Socio-Économiques Du Diabète Chez Les Diabétiques Suivis À Porto-Novo En 2016
}

\author{
Alassani Adébayo, \\ Mama Cisse Ibrahim,
}

Département de Médecine et Spécialités Médicales,

Faculté de Médecine, Université de Parakou, Bénin

Gninkoun Jules,

Département de Médecine et Spécialités Médicales,

Faculté des Sciences de la Santé, Université d'Abomey Calavi

Gounongbe Fabien,

Codjo Léopold,

Gandaho Prosper,

Département de Médecine et Spécialités Médicales,

Faculté de Médecine, Université de Parakou, Bénin

Doi:10.19044/esj.2020.v16n18p290 URL:http://dx.doi.org/10.19044/esj.2020.v16n18p290

\section{Résumé}

Le diabète est une affection qui pose un problème de santé publique avec des complications sur divers organes. Son impact socio-économique est peu étudié au Bénin. L'objectif de la présente étude était de décrire l'impact socio-économique du diabète chez les diabétiques suivis à l'hôpital universitaire de Porto-Novo en 2016. Il s'est agi d'une étude transversale et descriptive allant du 7 Novembre au 23 Décembre 2016. La population d'étude était constituée des diabétiques des deux sexes, âgés d'au moins 18 ans et ayant donné leur consentement. L'échantillonnage a été exhaustif. L'impact social et économique du diabète a été apprécié grâce à un questionnaire pré-établi. La saisie et l'analyse des données ont été faites par les logiciels EPI DATA 3.1, EPI INFO 7 et STATA 11. Au total, 133 diabétiques ont été recrutés pour l'étude. L'âge moyen est de $54 \pm 8$ ans. Il y avait une prédominance féminine avec un sexe ratio de 0,6 . Sur le plan social, $26,3 \%$ avaient une atténuation des relations avec le conjoint, 83,5\% n'arrivaient pas à remplir les besoins de la famille et $69,9 \%$ avaient une altération de la qualité de vie. Sur le plan économique, $94 \%$ avaient des absences répétées au travail, 68,4\% avaient diminué les heures de travail, $54,3 \%$ ont signalé une diminution de leur revenu mensuel et tous avaient signalé l'augmentation de leurs dépenses mensuelles. Le diabète a un impact 
socio-économique négatif important sur les diabétiques à Porto-Novo. La prise en charge des diabétiques devrait aborder ce volet.

Mots clés : Impact Socio-Économique, Diabète, Porto-Novo, Bénin

\title{
Social and Economic Impact of Diabetes in Diabetic Patients Followed in Porto-Novo in 2016
}

\author{
Alassani Adébayo, \\ Mama Cisse Ibrahim, \\ Département de Médecine et Spécialités Médicales, \\ Faculté de Médecine, Université de Parakou, Bénin \\ Gninkoun Jules, \\ Département de Médecine et Spécialités Médicales, \\ Faculté des Sciences de la Santé, Université d'Abomey Calavi \\ Gounongbe Fabien, \\ Codjo Léopold, \\ Gandaho Prosper, \\ Département de Médecine et Spécialités Médicales, \\ Faculté de Médecine, Université de Parakou, Bénin
}

\begin{abstract}
Diabetes is a disease that poses a public health problem with complications on various organs. Its socio-economic impact is little studied in Benin. The objective of this study was to describe the socio-economic impact of diabetes in diabetics followed in teaching hospital of Porto-Novo in 2016. This was a cross-sectional and descriptive study from November 7 to December 23, 2016. The study population consisted of diabetics of both sexes who have age at least 18 years old and have given their consent. The sampling was exhaustive. The social and economic impact of diabetes was assessed using a pre-established questionnaire. Data entry and analysis was done by software EPI DATA 3.1, EPI INFO 7 and STATA 11. A total of 133 diabetics were recruited for the study. The average age is $54 \pm 8$ years. The sex ratio $\mathrm{men} /$ women is 0.6 . On a social level, $26.3 \%$ had a weakening of relationships with the spouse, $83.5 \%$ could not meet the needs of the family and $69.9 \%$ had an impairment in the quality of life. Economically, $94 \%$ had repeated absences from work, $68.4 \%$ had reduced work hours, $54.3 \%$ reported a decrease in their
\end{abstract}


monthly income and all had reported an increase in their monthly expenses. Diabetes has a significant negative socio-economic impact on diabetics in Porto-Novo. The management of diabetics should address this aspect.

Keywords: Socio-Economic Impact, Diabetes, Porto-Novo, Benin

\section{Introduction}

Le diabète est une maladie chronique qui pose un problème de santé publique aussi bien dans les pays développés que dans les pays de développement. Selon les estimations de la Fédération Internationale du Diabète, 425 millions de personnes vivent avec le diabète avec une projection à 693 millions en 2045 si aucune mesure préventive n'est prise [Pedron S et al. 2019, Osuji NA et al. 2018]. Il s'agit d'une affection responsable de plusieurs complications largement étudiées. Le coût direct de la prise en charge du diabète et de ses complications est très important [Sheida $J$ et al. 2018]. Au plan mondial, 727 milliard de dollars ont été consacrés à la prise en charge du diabète avec une estimation à 776 milliard de dollars en 2045 [Pedron S et al. 2019]. En 2017, aux États-Unis, ce coût annuel est de 237 milliards de dollars [Hird TR et al. 2019]. Au Bénin en 2013, le coût annuel direct de la prise en charge du diabète variait entre 117000 et 455000 FCFA par individu; ce coût peut atteindre 1756000 FCFA en cas de complications [Alouki K et al. 2015]. En dehors du coût direct, le diabète est responsable de coût indirect. Aux États-Unis ce coût indirect a atteint 90 milliard de dollars en 2017 et est en rapport avec l'absentéisme au travail (3,3 milliard de dollars), la baisse de production (26,9 milliard de dollars), l'invalidité (37,5 milliard de dollars) et la mort prématurée (19,9 milliard de dollars) [American Diabetes Association. 2018]. Selon les résultats de plusieurs études, les personnes vivant avec le diabète cumulent plus de jours d'absence au travail, un risque d'invalidité plus élevé comparativement aux sujets non diabétiques [Breton $\mathrm{M}$ et al. 2013, Ervasti J et al. 2015, Lalluka T et al. 2016]. Au Bénin, il existe peu d'études qui ont abordé le coût indirect du diabète. Aucune donnée n'existe sur les modifications sociales observées chez les diabétiques ainsi que celles de son environnement de travail. L'objectif de la présente étude était de décrire l'impact socio-économique du diabète chez les diabétiques suivis à PortoNovo.

\section{Patients et méthodes}

L'étude a été effectuée dans le Centre Hospitalier Universitaire Départemental de l'Ouémé Plateaux à Porto-Novo (CHUP-OP) en République du Bénin.

Il agit d'une étude transversale et descriptive qui a couvert la période allant du 7 Novembre au 23 Décembre 2016. 
La population d'étude était constituée des diabétiques suivis au CHUD-OP. Les diabétiques dépistés depuis au moins 1 an, âgés d'au moins 18 ans et ayant donné leur consentement ont été inclus dans l'étude. Les diabétiques incapables de répondre et les gestantes n'ont pas été inclus dans l'étude. Un recensement exhaustif des diabétiques a été fait. Les impacts socio-économiques du diabète ont été décrits. Il était question de recenser les modifications sociales et économiques observées depuis le dépistage du diabète. Ces modifications peuvent être selon le cas être inchangées, diminuées ou renforcées. Les modifications sociales avaient concerné la situation matrimoniale, les relations avec le ou la conjointe, les enfants, les collègues ou les camarades, l'assurance des charges familiales, la qualité de vie et l'état émotionnel. La dépression était appréciée par l'échelle de "La Hospital Anxiety and Depression" [Snaith RP et al. 1983]. Celles économiques avaient concerné l'assiduité au travail, la qualité du travail, les dépenses et revenus mensuels.

La collecte des données a été faite par l'entrevue face à face. Une fiche de collecte de données avait été utilisée.

Le traitement et l'analyse des données ont été saisies à l'aide du logiciel EPIDATA version 3.1 après vérification de chaque fiche, elles ont été analysées à l'aide des logiciels EPIINFO version 7 et STATA 11.

La confidentialité des données était respectée lors de l'enquête. Après avoir expliqué aux diabétiques le but du travail, ils étaient libres de participer ou de ne pas participer à l'enquête et qu'une fois qu'ils ont accepté. Ils étaient en droit de désister après. L'anonymat était requis sur les fiches d'enquête. L'autorisation des responsables du CHUP-OP a été obtenue.

\section{Résultats}

\section{Caractéristiques générales de la population d'étude}

Les caractéristiques générales de la population d'étude figurent dans le Tableau I. Au total 133 patients diabétiques ont été inclus dans l'étude. Une prédominance féminine $(62,4 \%)$ a été observée avec un sex-ratio de 0,6 . L'âge moyen des patients était de 54,9 $\pm 8,67$ ans avec des extrêmes de 35 et 74 ans. La plupart des patients $(92,50 \%)$ avaient un âge compris entre 40 et 70 ans. Les patients non instruits représentaient $18,8 \%$; près de la moitié (49\%) étaient des commerçants; la plupart $(98,50 \%)$ étaient mariés. Le nombre d'enfants à charge était supérieur ou égal à 5 dans $97 \%$ des cas avec une

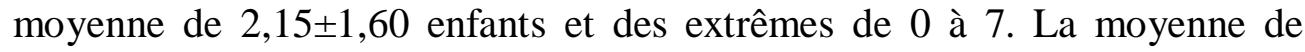
l'ancienneté de dépistage du diabète était de 7,90土7,76 ans avec des extrêmes de 1 à 29 ans. 
Tableau I: Caractéristiques générales des sujets diabétiques suivis à Porto-Novo en 2016

\begin{tabular}{|c|c|c|}
\hline & $\mathbf{N}$ & $\%$ \\
\hline \multicolumn{3}{|l|}{ Sexe } \\
\hline Masculin & 50 & 37,60 \\
\hline Femmes & 83 & 62,40 \\
\hline \multicolumn{3}{|l|}{ Age (ans) } \\
\hline$\leq 40$ & 6 & 4,50 \\
\hline $40-70$ & 123 & 92,50 \\
\hline$>70$ & 4 & 3,00 \\
\hline \multicolumn{3}{|c|}{ Niveau d'instruction } \\
\hline Aucun & 25 & 18,80 \\
\hline Primaire & 39 & 29,30 \\
\hline Secondaire & 42 & 31,60 \\
\hline Supérieur & 27 & 20,30 \\
\hline \multicolumn{3}{|l|}{ Profession } \\
\hline Fonctionnaires & 40 & 30,00 \\
\hline Artisans & 28 & 21,00 \\
\hline Commerçants & 65 & 49 \\
\hline \multicolumn{3}{|c|}{$\begin{array}{l}\text { Situation matrimoniale avant le diagnostic } \\
\text { du diabète }\end{array}$} \\
\hline Célibataires & 2 & 1,50 \\
\hline Mariés & 131 & 98,50 \\
\hline \multicolumn{3}{|c|}{ Enfants à charge } \\
\hline$\leq 5$ & 129 & 97 \\
\hline$>5$ & 4 & 3 \\
\hline \multicolumn{3}{|c|}{ Ancienneté de dépistage du diabète (ans) } \\
\hline$\leq 5$ & 41 & 30,80 \\
\hline $5-10$ & 68 & 51,10 \\
\hline$>10$ & 24 & 18,10 \\
\hline
\end{tabular}

\section{Description de l'impact social du diabète}

La description de l'impact social du diabète figure dans le tableau II. La situation matrimoniale des patients n'avait pas changé. Les relations avec le conjoint et les enfants ont été atténuées respectivement chez $26,30 \%$ et 2,30 $\%$ des patients. Les relations avec les collègues et ou camarades étaient atténuées chez $6 \%$ des diabétiques. Les relations abordées ont concerné celles fraternelles, amicales et ou amoureuses (cas du conjoint ou conjointe). Les besoins de la famille ne se réalisaient pas chez $83,50 \%$ des patients. La qualité de vie personnelle et celle avec l'entourage était altérée respectivement chez $69,90 \%$ et $35,30 \%$ des patients. La plupart $(92,48 \%)$ des patients avaient un état émotionnel dépressif. 
Tableau II : Description de l'impact social du diabète chez les diabétiques suivis à PortoNovo en $2016(n=133)$

\begin{tabular}{|c|c|c|}
\hline & $\mathbf{N}$ & $\%$ \\
\hline \multicolumn{3}{|c|}{ Situation matrimoniale } \\
\hline Inchangée & 133 & 100 \\
\hline \multicolumn{3}{|c|}{ Relations avec le conjoint } \\
\hline Inchangées & 78 & 58,70 \\
\hline Renforcées & 20 & 15 \\
\hline Atténuées & 35 & 26,30 \\
\hline \multicolumn{3}{|c|}{ Relations avec les enfants } \\
\hline Inchangées & 78 & 58,70 \\
\hline Renforcées & 52 & 39,10 \\
\hline Atténuées & 3 & 2,20 \\
\hline \multicolumn{3}{|c|}{ Accomplissement des besoins de la famille } \\
\hline Oui & 22 & 16,50 \\
\hline Non & 111 & 83,50 \\
\hline \multicolumn{3}{|c|}{ Relations avec les collègues ou camarades } \\
\hline Inchangées & 125 & 94 \\
\hline Atténuées & 8 & 6 \\
\hline \multicolumn{3}{|c|}{ Qualité de vie personnelle } \\
\hline Inchangée & 40 & 30,10 \\
\hline Altérée & 93 & 69,90 \\
\hline \multicolumn{3}{|c|}{ Qualité de vie de l'entourage } \\
\hline Inchangée & 86 & 64,70 \\
\hline Altérée & 47 & 35,30 \\
\hline \multicolumn{3}{|c|}{ Etat émotionnel } \\
\hline Inchangé & 10 & 7,5 \\
\hline Dépressif & 123 & 92,50 \\
\hline
\end{tabular}

\section{Description de l'impact économique}

La description de l'impact économique du diabète figure dans le tableau III. La plupart (94\%) des patients avaient des absences répétées au travail. L'assiduité au travail était atténuée chez $66,20 \%$ des diabétiques. Environ 7 patients sur $10(68,40 \%)$ avaient diminué le nombre d'heures de travail. Dans près de la moitié $(48,90 \%)$ des cas, la qualité du travail était altérée. Le revenu mensuel avait diminué chez $53,40 \%$ des patients et les dépenses étaient augmentées chez tous les diabétiques. Le niveau de revenu mensuel a concerné les gains mensuels des patients à travers leurs activités tandis que les dépenses sont relatives à celles nécessaires à l'accomplissement des besoins fondamentaux y compris la santé. 
Tableau III : Description de l'impact économique du diabète chez les diabétiques suivis à Porto-Novo en 2016 ( $n=133)$

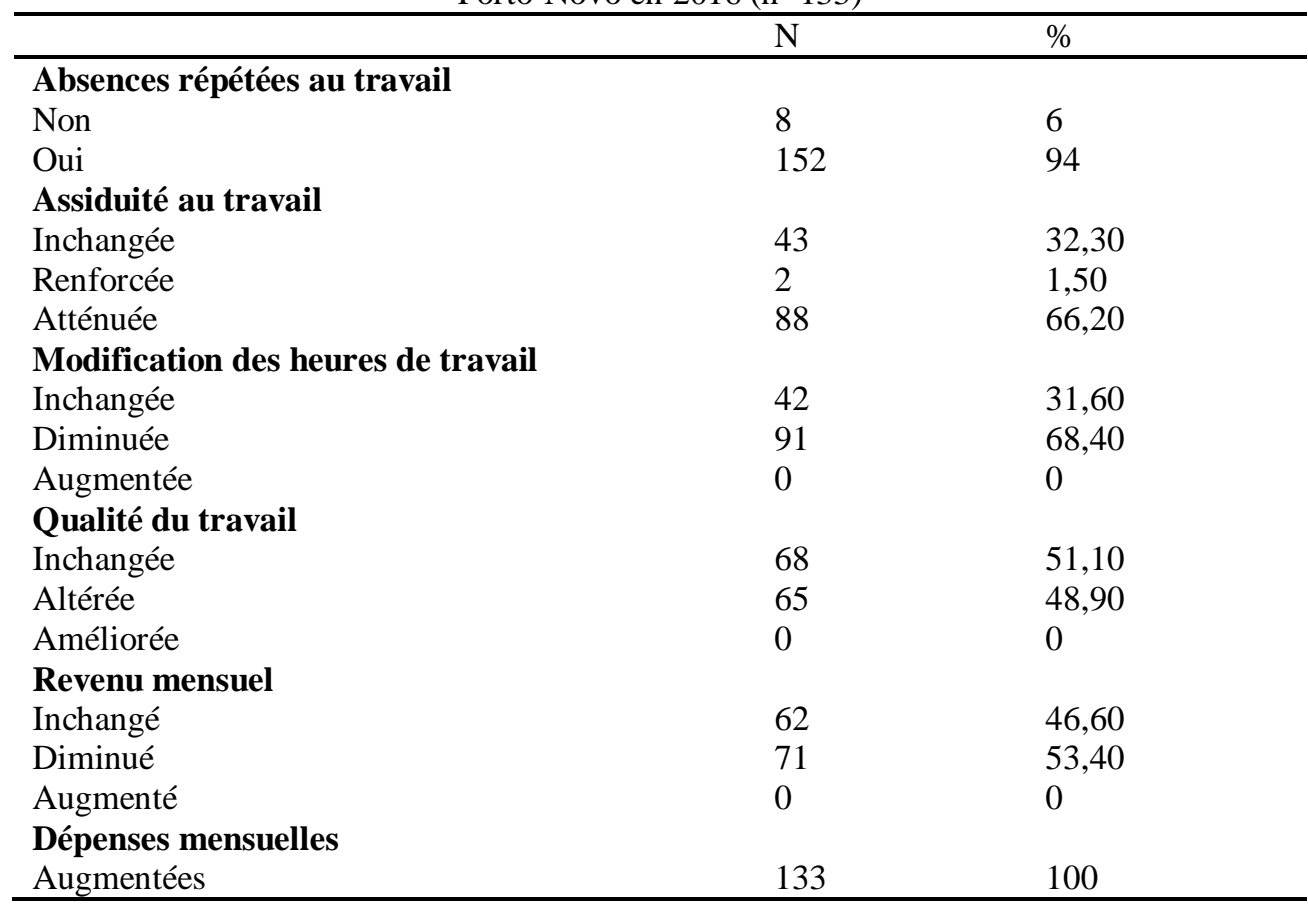

\section{Discussion}

La présente étude est l'une des rares au Bénin à s'intéresser à l'impact socio-économique du diabète. Au terme de cette étude, les modifications sociales et économiques observées après le dépistage du diabète ont été recensées. L'étude a inclus seulement 133 patients diabétiques et a été menée à l'hôpital universitaire de Porto-Novo. Les résultats issus de cette étude ne peuvent donc pas se généraliser à toute la population des diabétiques du Bénin mais donnent une idée des modifications familiales et relatives au travail observées chez ces derniers.

L'impact social du diabète était caractérisé par l'atténuation des relations avec le conjoint et les enfants respectivement chez $26,3 \%$ et $2,3 \%$ des patients, avec les collègues et ou camarades $6 \%$ des diabétiques. L'accomplissement des besoins de la famille ne se réalisait pas chez $83,5 \%$ des patients. La qualité de vie personnelle et celle avec l'entourage est altérée respectivement chez $69,9 \%$ et $35,3 \%$ des patients. La plupart $(92,48 \%)$ des patients ont un état émotionnel dépressif. Ceci montre les difficultés d'insertion sociale éprouvées par les diabétiques. Des résultats similaires étaient rapportés dans l'étude de Oyewole et al. [Oyewole $O$ et al. 2019] où la moitié $(50,6 \%)$ des diabétiques avaient des difficultés à assurer les charges familiales, 35,2 \% avaient perdu les relations avec les amis, 24,7 \% étaient incapables de dresser le lit, 25,9\% n'arrivaient pas à se laver seuls et $63 \%$ 
étaient déprimés. Dans l'étude de Ramkisson et al. [Ramkisson S et al. 2017], $22 \%$ des diabétiques ne sont acceptés par leurs parents ou amis, $24 \%$ ne sont pas encouragés par leurs parents et $40 \%$ ne sont pas écoutés par leur entourage. L'altération de la qualité de la vie de couple des diabétiques et la mauvaise qualité de vie des parents des enfants diabétiques avaient été respectivement rapportées par Thorsteinsson et al. [Thorsteinsson E et al. 2017] et Wiebe et al. [Wiebe D et al. 2016]. Dans l'étude de Pesantes et al. [Pesantes M et al. 2020] au Mozambique, des femmes diabétiques avaient été abandonnées par leur mari et certains hommes diabétiques ont des difficultés à avoir des relations sexuelles du fait des complications et de l'asthénie. Le même auteur rapporte dans une autre étude les difficultés relationnelles avec les parents que rencontrent les diabétiques qui sont incapables de s'assoir sur la même table à cause de la différence quantitative et surtout qualité de leur alimentation [Pesantes $M$ et al. 2018].

Concernant l'impact économique, la plupart (94\%) des patients avaient des absences répétées au travail, l'assiduité au travail était atténuée chez $66,2 \%$ des diabétiques, $68,4 \%$ avaient diminué le nombre d'heures de travail, 48,9\% avaient une qualité du travail altérée, le revenu mensuel avait diminué chez 53,4 \% des patients et les dépenses étaient augmentées chez tous les diabétiques. Ceci a pour conséquence une diminution des revenus des patients alors que leurs dépenses se trouvent augmentées. Ces différents impacts négatifs du diabète sur les patients avaient été rapportés par d'autres autres. C'est le cas des études de Mutambudzi et al. [Mutambudzi M et al. 2019] et de Nexø et al. [Nexø MA et al. 2019], qui avaient rapporté que les diabétiques passaient moins d'heures au travail et étaient souvent absents à cause de leur maladie. Selon Breton et al. [Breton M et al. 2013], les diabétiques perdaient 26,3 jours de travail par an comparativement à 12 jours chez les sujets non diabétiques. Au cours du travail les diabétiques éprouvent des difficultés : $46 \%$ étaient incapables de rester debout pendant plus de 30 minutes, 38,3 étaient des difficultés de concentration pendant 10 minutes et $61,1 \%$ étaient incapables de marcher sur $1 \mathrm{~km}$ [Oyewole O et al. 2019].

\section{Conclusion}

Le diabète a entraîné des modifications sociales et économiques néfastes non seulement pour les personnes atteintes mais aussi leur entourage. La prise en charge des diabétiques doit être globale et prendre en compte leur environnement socio-économique. Le soutien de la famille et une modification des lois du travail prenant en compte les difficultés socio-économiques des diabétiques s'avèrent nécessaires. 


\section{References:}

1. Pedron S, Emmert-Fees K, Laxy M, Schwettmann L. (2019) The impact of diabetes on labour market participation: a systematic review of results and methods. BMC Public Health; 19(1):25-37

2. Osuji NA, Ojo OS, Malomo SO, Sogunle PT, Egunjobi AO, Odebunmi O. (2018) Relationship between glycemic control and perceived family support among people with type 2 diabetes mellitus seen in a rich kinship network in Southwest Nigeria. Family Medicine and Community Health; 6(4):168-77

3. Sheida J, Sorur J, Zhaleh N. (2019) The Relationship between Emotional Intelligence and Self-Efficacy in Type II Diabetes Patients. Iranian Journal of Diabetes and Obesity; 11(2):106-11

4. Hird TR, Zomer E, Owen A, Chen L, Ademi Z, Magliano DL. (2019) The impact of diabetes on productivity in China. Diabetologia; 62:1195-203

5. Alouki K, Delisle H, Besançon S, Baldé N, Sidibé-Traoré A, Drabo J. (2015) Simple calculator to estimate the medical cost of diabetes in sub-Saharan Africa World J Diabetes; 6(16):1312-22

6. American Diabetes Association. (2018) Economic Costs of Diabetes in the U.S. in 2017. Diabetes Care; 41:917-28

7. Breton M, Guénette L, Amichie M, Kayibanda J, Grégoire J, Moisan J. (2013) Burden of Diabetes on the Ability to Work. Diabetes Care; 36:740-9

8. Ervasti J, Virtanen M, Penti J, Lalluka T, Tinghög P, Kjeldgard L. (2015) Work Disability Before and After Diabetes Diagnosis: A Nationwide Population-Based Register Study in Sweden. Public Health; 105:22-9

9. Lallukka T, Ervasti J, Rutz-Mittendorfer E, Tinghög P, Kjeldgard L, Pentti J. (2016) The joint contribution of diabetes and work disability to premature death during working age: a population-based study in Sweden. Scandinavian Journal of Public Health; 44:580-6

10. Snaith RP, Zigmond AS. (1983) The Hospital Anxiety And Depression Scale. Acta Psychiatr Scand.; 67(6):361-70.

11. Oyewole O, Odusan O, Ale A. (2019) Global disability burden and its predictors among adult Nigerians living with Type-2 diabetes. Ghana Med J; 53(2):135-41

12. Ramkisson S, Pillay BJ, Sibanda W. (2017) Social support and coping in adults with type 2 diabetes. Afr J Prm Health Care Fam Med.;9(1) : 1-8

13. Thorsteinsson E, Loi N, Rayner K. (2017) Self-efficacy, relationship satisfaction, and social support: the quality of life of maternal caregivers of children with type 1 diabetes. Peer J; 1(2):1-16 
14. Wiebe D, helgeson V, Berg C. (2016) The Social Context of Managing Diabetes across the Life Span Am Psychol; 71(7):526-38

15. Pesantes M, Somerville C, Singh S, Perez-Leon S, Madede T, Suggs S. (2020) Disruption, changes, and adaptation: Experiences with chronic conditions in Mozambique, Nepal and Peru. Global Public Health; 15 (3): 372-83

16. Pesantes M, Valle A, Diez-Canseco F, Bernabé-Ortiz, Portocarrero J, Trujillo A. (2018) Family Support and Diabetes: Patient's Experiences From a Public Hospital in Peru Qualitative Health Research; 28(12):1871-82

17. Mutambudzi M, Gonzalez C, Wong R. (2019) Impact of Diabetes and Disease Duration on Work Status Among U.S. Older Adults. Journal of Aging and Health; 1-19

18. Nexø, MA, Pedersen J, Cleal B, Bjorner, J. (2019) Increased risk of long term sickness absence, lower rate of return to work and higher risk of disability pension among people with type 1 and type 2 diabetes mellitus: a Danish retrospective cohort study with up to 17 years' follow up. Diabetic Medicine; 1(2):1-7 